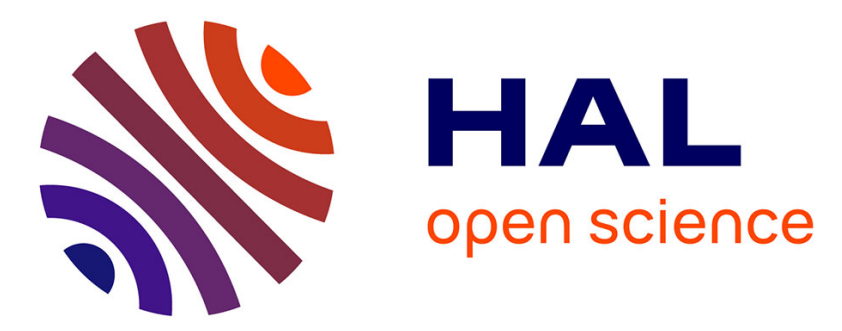

\title{
New integral representations in the dynamic uncoupled thermoelasticity
}

Victor Sheremet, Guy Bonnet, T. Speianu

\section{To cite this version:}

Victor Sheremet, Guy Bonnet, T. Speianu. New integral representations in the dynamic uncoupled thermoelasticity. Journal of Thermal Stresses, 2009, 32 (10), pp.1043-1064. 10.1080/01495730903103119. hal-00691019

\section{HAL Id: hal-00691019 \\ https://hal.science/hal-00691019}

Submitted on 19 Jan 2016

HAL is a multi-disciplinary open access archive for the deposit and dissemination of scientific research documents, whether they are published or not. The documents may come from teaching and research institutions in France or abroad, or from public or private research centers.
L'archive ouverte pluridisciplinaire HAL, est destinée au dépôt et à la diffusion de documents scientifiques de niveau recherche, publiés ou non, émanant des établissements d'enseignement et de recherche français ou étrangers, des laboratoires publics ou privés. 


\title{
NEW INTEGRAL REPRESENTATIONS IN THE DYNAMIC UNCOUPLED THERMOELASTICITY
}

\author{
Victor Sheremet $^{1}$, Guy Bonnet ${ }^{2}$, and Tatiana Speianu ${ }^{3}$
}

${ }^{1}$ Department of Engineering and Mathematics Sciences, Agrarian State University of Moldova, Chisinau, Moldova

${ }^{2}$ Laboratoire de Modélisation et Simulation Multi-Echelle FRE 3160 CNRS,

Université Paris Est, 77454 Marne la Vallée Cedex, France

${ }^{3}$ Department of Mathematics, Agrarian State University of Moldova,

Chisinau, Moldova

New integral representations of homogeneous $3 D$ uncoupled dynamic thermoelasticity for semi-infinite cylindrical domains with curvilinear surfaces placed at infinity and subject to mixed boundary conditions on the plane boundaries are obtained. The representations are given in the form of integral convolutions involving a Green's function for the parabolic heat conduction equation, as well as Green's function for the isothermal elastodynamics. A multi-integral representation of solution to a particular initial-boundary value problem for an infinite wedge is included.

Keywords: Canonical cylindrical domains; Green's integral formula; Heat flux; Heat source; Influence functions; Temperature; Theorem on dilatation; Wedges

\section{INTRODUCTION}

Green's function plays a leading role in finding solutions in integrals for boundary value problems (BVP) in the different fields of mathematical physics. The theory of thermoelasticity, which is a synthesis of the theory of heat conduction and the theory of elasticity, is one of such fields. By our days a number of theories of thermoelasticity have been developed and described in literature [1-6]. But many new developments of thermoelasticity, and many references are included in the book [7]. The best developed theory, which is widely used in practical calculations, is the theory of thermal stresses, i.e. the theory of uncoupled thermoelasticity, when the temperature field does not depend on the field of elastic displacements. The advantages that the solutions of BVP of thermoelasticity have in the form

One of the authors, Dr V. Seremet, is grateful to the University Paris-Est, Marne-la-Vallee, France, for the support of his two research visits to that university. Also he expresses many thanks to the reviewer of this paper, whose comments have contributed substantially to its improvements.

Address correspondence to Victor Sheremet, Department of Engineering and Mathematics Sciences, Agrarian State University of Moldova, Mircesti Street 44, Chisinau 2049, Moldova. E-mail: v.seremet@uasm.md 
of integrals are well known. In the theory of thermal stresses, the following observations are worth mentioning. In the theory of uncoupled heat conduction, that is, a constitutional part of the theory of thermal stresses, to solve a BVP, a Green's integral formula provides the temperature field resulted from a thermal exposure.

The analogous Green's integral formula determines the field of elastic displacements produced by the known mechanical actions. In the heat conduction problems and in the linear dynamic elasticity problems the integral formulas include the initial data, inner heat sources, boundary temperature, body forces and boundary tractions. In the case of the integral Maysel's formula, the desired solution (the thermoelastic displacements) is not represented directly in terms of the given data, but in terms of a temperature field, which is to be found. This fact introduces certain inconvenience in application of Maysel's formula except for the case when the temperature field is known a priori. Hence, to obtain Green's integral formula it is necessary to construct either Green's functions or Green's tensors. To obtain the integral Maysel's formula in uncoupled dynamic thermoelasticity, a twostage procedure is applied. First, we find a temperature field, and in the second stage we construct a function of influence corresponding to a unit body force and representing a volume dilatation.

Finding the influence function in the second stage is not a trivial problem. This is why obtaining such a function, especially in a closed form, is highly appreciated by the specialists in the field. So, for some particular BVP's such influence functions have been obtained in [8-11], and the associated integral formulae for thermoelastic displacements have been found. For static thermoelasticity the integral representations are obtained in [9-12]. The general influence function formulas corresponding to an inner point heat source, or to a unit point temperature, or a unit point heat flux on a boundary in uncoupled dynamical thermoelasticity have been obtained in [13, 14]. In [13], a generalization of Maysel's and Green's integral formulae onto dynamical uncoupled thermoelasticity is obtained for domains, the surfaces of which are planes parallel to the Cartesian coordinate planes. Analogical results in static thermoelasticity, for a number of boundary conditions have been obtained in [15]. The results obtained in the Cartesian coordinates can be used to derive Poisson's type integrals of uncoupled dynamic thermoelasticity.

In this paper, new integral representations for a wide class of BVP's in uncoupled dynamical thermoelasticity in the cylindrical system of coordinates is obtained. This class of problems deals with the canonical cylindrical (polar) domains, whose surfaces (boundaries) are planes (or straight lines) on which particular mixed boundary conditions are prescribed, but the curvilinear surfaces (boundaries) are placed at infinity. To obtain the results, a volume dilatation theorem of isothermal elastodynamics is proved. The general integral formulae for influence functions as well as for the general Green's type integral formula obtained in [13-15] are used to obtain the results in cylindrical system of coordinates. As an example, Green's type integral formula for displacements for a particular initial boundary value problem of uncoupled dynamical thermoelasticity for an infinite wedge is obtained. 


\section{FORMULATION OF THE PROBLEMS AND THE WAYS TO SOLVE THEM}

The main objectives of this paper are:

1. To obtain the integral influence function formulae for a wide class of particular BVP's of uncoupled dynamical thermoelasticity for so-called canonical cylindrical domains. By a canonical cylindrical domain we mean a domain, whose surfaces are the coordinate planes with equations $\Gamma_{z l}(0 \leq r<\infty ; 0 \leq$ $\left.\varphi \leq \alpha_{n} ; z=a_{l}\right)$ and/or $\Gamma_{\varphi n} ;\left(0 \leq r<\infty ; \varphi=\alpha_{n} ; 0 \leq z \leq a_{l}\right) ; l, n=0$, and/or $l, n=1$ - in cylindrical system of coordinates $(r, \varphi, z)$ and coordinate lines $\Gamma_{\varphi n}\left(0 \leq r<\infty ; \varphi=\alpha_{n}\right)$ - in polar system of coordinates $(r, \varphi)$, where $0 \leq \alpha_{n} \leq$ $2 \pi ; a_{l} \geq 0$. The curvilinear surfaces (boundaries) of this kind of cylindrical (polar) domain are placed at infinity. As examples of such domains are:

a. space, half-space, quarter of space, eighth part of space or octant, layer, semi-layer, quarter of layer, wedges, semi-wedges with the different angles $\alpha$, including $0<\alpha=\pi / n \leq \pi / 2 ; n=3,4,5, \ldots$, ;

b. Plane, half-plane, quarter of plane (quadrant) and also different plane wedges, it means the canonical polar domains described by equations $0 \leq r<\infty ; 0 \leq$ $\varphi \leq \alpha ; 0<\alpha \leq 2 \pi$. By an influence function we mean a solution to a particular initial boundary value problem of uncoupled dynamic thermoelasticity corresponding to an inner unit point heat source, or a unit point temperature or a unit point heat flux on the boundary.

2. To obtain the influence functions in explicit form and the closed form solution in a form of Green's type integrals for a mixed BVP in uncoupled dynamical thermoelasticity for an infinite wedge with the angles $\alpha=\pi / n ; n=2,3,4, \ldots$, .

To this end, we transform the results for Cartesian coordinates obtained in [13-15] onto the cylindrical ones. Next, we use the following steps.

1. We determine the functions of influence corresponding to an inner unit concentrated force and representing a dilatation of isothermal elastodynamics. To achieve this, it is sufficient to use the integral representations for a volume dilatation via the respective Green's function.

2. We formulate a parabolic heat conduction problem to derive the desired influence function corresponding to an inner unit point heat source.

3. We introduce the derived volume dilatation and Green's function for temperature in the general integral formula for the function of influence corresponding to an inner unit point heat source of uncoupled dynamic thermoelasticity and obtain the desired integral formula. The integral formula is to be presented in a form of the convolution over time and volume of product of two Green's functions. One of the Green's functions corresponds to a parabolic heat conduction problem while the other is the Green's dilatation function corresponding to a problem of isothermal elastodynamics.

To obtain an explicit form of the influence functions and of Green's type integral formula for displacements for a mixed BVP of uncoupled dynamical thermoelasticity for a wedge with the angles $\alpha=\pi / n ; n=2,3,4, \ldots$, we follow the 
following steps:

a. We find an analytical expression for Green's function that satisfies a nonstationary heat conduction problem for the wedge.

b. We find an analytical expression for the influence functions that satisfy a volume dilatation initial boundary value problem of isothermal elastodynamics for the wedge.

c. We compute a convolution over time and volume of the product of the analytical expressions from points

a. and $b$. to obtain the desired result.

d. We derive the Green's type integral formula that represents a solution to an initial boundary value problem of uncoupled dynamic thermoelasticity for the wedge.

\section{INFLUENCE FUNCTIONS AND GREEN'S TYPE FORMULA IN DYNAMICAL UNCOUPLED THERMOELASTICITY}

\section{The Basic Equations of Dynamical Thermoelasticity in Cylindrical Coordinates}

In this section, we recall the formulae for influence functions and for the general Green's type integral formula, as well as the necessary equations to determine them, obtained before in Cartesian system of coordinates in [13]. These results are rewritten in the cylindrical system of coordinates. The influence functions are given in the form of a convolution over the body volume $V$ and the time $t$ of the two Green's functions. The first is the Green's function $G(M, \bar{N} ; t-s)$ for an initial-boundary value heat conduction problem. The second is a function of influence corresponding to a unit point temperature in a BVP of elastodynamics. The second influence function represents a volume dilatation $\Theta^{(q)}(\bar{N}, N ; \varsigma-\tau),(s \equiv$ $r, \varphi, z)$ of elastodynamics corresponding to a unit concentrated body force. Thus, the displacement corresponding to an inner unit point heat source $F(M, \tau)=\delta(M-$ $N) \delta(t-\tau)$ and representing a solution to an initial-boundary value problem of dynamic uncoupled thermoelasticity is determined by the following integral formula

$$
U_{q}(M, N ; t-\tau)=\gamma \int_{0}^{t} d \varsigma \int_{V} G(M, \bar{N} ; t-\varsigma) \Theta^{(q)}(\bar{N}, N ; \varsigma-\tau) d V(\bar{N})
$$

where $(q \equiv \rho, \psi, \xi) ; \gamma=\alpha_{t}(2 \mu+3 \lambda)$ is the thermoelastic constant; $\alpha_{t}$ is the coefficient of the linear thermal expansion; $\lambda, \mu$ are Lame's constants of elasticity. Unlike traditional Green's functions, the introduced influence functions $U_{q}(M, N ; t-\tau)$ do not possess the symmetry.

Note that the main difficulties in solving a BVP of dynamical uncoupled thermoelasticity, using Eq. (1), consist in:

(1) Constructing the influence function corresponding to a unit concentrated body force and representing a volume dilatation $\Theta^{(q)}(\bar{N}, N ; \varsigma-\tau)$ of elastodynamics.

(2) Constructing Green's function $G(M, \bar{N} ; t-\varsigma)$ for an initial boundary value heat conduction problem.

(3) Calculating the integrals in Eq. (1) to determine the influence functions $U_{q}(M, N ; t-\tau)$. 
So, to construct the functions $\Theta^{(q)}(M, N ; \varsigma-\tau)$ we need to solve the initial boundary value problem of elastodynamics which is described by:

(a) the displacement equations of elastodynamics in terms of the Green's tensor components $U_{S}^{(q)}$ :

$$
\begin{aligned}
& \mu\left(\nabla_{M}^{2} U_{r}^{(q)}-\frac{1}{\rho^{2}} U_{r}^{(q)}-\frac{2}{r^{2}} \frac{\partial U_{r}^{(q)}}{\partial \varphi}\right)+(\lambda+\mu) \frac{\partial \Theta^{(q)}}{\partial r} \\
& \quad-\rho_{0} \frac{\partial^{2}}{\partial t^{2}} U_{r}^{(q)}+\delta_{r q} \delta(M-N) \delta(t-\tau)=0 \\
& \mu\left(\nabla_{M}^{2} U_{\varphi}^{(q)}-\frac{1}{r^{2}} U_{\varphi}^{(q)}+\frac{2}{r^{2}} \frac{\partial U_{\rho}^{(q)}}{\partial \varphi}\right)+(\lambda+\mu) \frac{1}{r} \frac{\partial \Theta^{(q)}}{\partial \varphi} \\
& \quad-\rho_{0} \frac{\partial^{2}}{\partial t^{2}} U_{\varphi}^{(q)}+\delta_{\varphi q} \delta(M-N) \delta(t-\tau)=0 \\
& \mu \nabla_{M}^{2} U_{z}^{(q)}+(\lambda+\mu) \frac{\partial \Theta(q)}{\partial z}-\rho_{0} \frac{\partial^{2}}{\partial t^{2}} U_{z}^{(q)}+\delta_{z q} \delta(M-N) \delta(t-\tau)=0
\end{aligned}
$$

(b) the homogeneous initial conditions

$$
U_{S}^{(q)}(M, N ; t-\tau=0)=0 ; \quad \frac{\partial U_{S}^{(q)}(M, N ; t-\tau=0)}{\partial t}=0
$$

written at an inner point $M \in V$, and

(c) the homogeneous boundary conditions

$$
\begin{aligned}
& U_{S}^{(q)}(\bar{M}, N ; t-\tau)=0 ; \quad \bar{M} \in \Gamma_{U} ; \quad P_{S}^{(q)}(\bar{M}, N ; t-\tau)=0 ; \quad P_{S}^{(q)}=\sigma_{S l}^{(q)} n_{l} ; \\
& \bar{M} \in \Gamma_{P} \quad \beta_{1} U_{S}^{(q)}(\bar{M}, N ; t-\tau)+\beta_{2} P_{S}^{(q)}(\bar{M}, N ; t-\tau)=0 ; \quad \bar{M} \in \Gamma_{U P}
\end{aligned}
$$

written at points $\bar{M}$ of the surface $\Gamma \equiv \Gamma_{U}+\Gamma_{P}+\Gamma_{U P}$. In Eq. (4), $\Gamma_{U}, \Gamma_{P}$ and $\Gamma_{U P}$ denote the surfaces on which the boundary conditions for displacements, tractions, or mixed (for displacements and tractions at the same points, but in different directions) are prescribed; $\rho_{0}$ is the density of the body; $\delta_{q l}$ is Kronecker's symbol.

According to point (2), to construct Green's function $G(M, \bar{N} ; t-\varsigma)$ we need to solve an initial boundary value heat conduction problem described by:

(a) the non-stationary heat conduction equation

$$
\begin{aligned}
& \left(\frac{\partial}{\partial t}-a \nabla_{M}^{2}\right) G(M, N ; t-\tau)=\delta(M-N) \delta(t-\tau) ; \\
& \quad M, N \in V, M \equiv(r, \varphi, z) ; \quad N \equiv(\rho, \psi, \xi) ; \quad t, \tau \geq 0, t>\tau
\end{aligned}
$$

(b) the initial homogeneous conditions, written at an inner point $M \in V$,

$$
\left.G(M, N ; t-\tau)\right|_{t-\tau=0}=0
$$


(c) the homogeneous boundary conditions

$$
\begin{aligned}
& G(\widetilde{M}, N ; t-\tau)=0 ; \quad \widetilde{M} \in \Gamma_{D} ; \quad \partial G(\widetilde{M}, N ; t-\tau) / \partial n_{\tilde{M}}=0 ; \quad \widetilde{M} \in \Gamma_{N} \\
& \left(\alpha \frac{\partial}{\partial n_{\bar{M}}}+a\right)(\tilde{M}, N ; t-\tau)=0 ; \quad \widetilde{M} \equiv(\tilde{r}, \tilde{\varphi}, \tilde{z}) \in \Gamma_{M}
\end{aligned}
$$

written at points $\widetilde{M}$ of surface $\Gamma \equiv \Gamma_{D}+\Gamma_{N}+\Gamma_{M}$. In Eq. (7), $\Gamma_{D}, \Gamma_{N}$ and $\Gamma_{M}$ denote the surfaces on which the boundary conditions of Dirichlet's, Neumann's, or mixed type (mixed boundary conditions describing the heat exchange with the external medium) are prescribed. In Eqs. (5) and (7) $\alpha$ is the coefficient of convective heat conductivity; $a$ is the coefficient of temperature conductivity.

Clearly, the influence functions $U_{q}(M, N ; t-\tau)$ represent an influence of the internal unit point heat source onto the desired dynamical thermoelastic displacements [13-15]. This is why the influence functions $U_{q}(M, N ; t-\tau)$ are expressed in terms of both the thermal and the mechanical fields.

\section{Boundary Value Problem that Determines the Influence Functions}

As in the Cartesian system of coordinates [13] the influence functions $U_{q}(M, N ; t-\tau)$ corresponding to a unit heat source $F(\bar{N}, \tau)=\delta(M-N) \delta(t-\tau)$ satisfy at a point $N \equiv(\rho, \psi, \xi)$ the equations of dynamic uncoupled thermoelasticity

$$
\begin{aligned}
& \mu\left(\nabla_{N}^{2} U_{\rho}-\frac{U_{\rho}}{\rho^{2}}-\frac{2}{\rho^{2}} \frac{\partial U_{\psi}}{\partial \psi}\right)+(\lambda+\mu) \frac{\partial \Theta}{\partial \rho}-\rho_{0} \frac{\partial^{2} U_{\rho}}{\partial \tau^{2}}-\gamma \frac{\partial G}{\partial \rho}=0 \\
& \mu\left(\nabla_{N}^{2} U_{\psi}-\frac{U_{\psi}}{\rho^{2}}+\frac{2}{\rho^{2}} \frac{\partial U_{\rho}}{\partial \psi}\right)+(\lambda+\mu) \frac{1}{\rho} \frac{\partial \Theta}{\partial \psi}-\rho_{0} \frac{\partial^{2} U_{\psi}}{\partial \tau^{2}}-\gamma \frac{1}{\rho} \frac{\partial G}{\partial \psi}=0 \\
& \mu \nabla_{N}^{2} U_{\xi}+(\lambda+\mu) \frac{\partial \Theta}{\partial \xi}-\rho_{0} \frac{\partial^{2} U_{\xi}}{\partial \tau^{2}}-\gamma \frac{\partial G}{\partial \xi}=0
\end{aligned}
$$

subject to the homogeneous mechanical initial and boundary conditions of the type presented in Eqs. (3) and (4). In Eq. (8), $G=G(M, N ; t-\tau)$ represents Green's function for the heat conduction problem (5)-(7). With respect to the variables $M \equiv(r, \varphi, z)$, the influence functions $U_{q}(M, N ; t-\tau)$ satisfy the following heat conduction equation

$$
\begin{aligned}
& \left(\frac{\partial}{\partial t}-a \nabla_{M}^{2}\right) U_{q}(M, N ; t-\tau)=\gamma \Theta^{(q)}(M, N ; t-\tau) ; \\
& \quad M, N \in V, M \equiv(r, \varphi, z) ; \quad N \equiv(\rho, \psi, \xi) ; \quad t, \tau \geq 0, t>\tau
\end{aligned}
$$

subject to the respective initial and boundary conditions as in Eqs. (6) and (7).

In the following section, the functions $U_{q}(M, N ; t-\tau)$ are to be called the main influence functions, because the other influence functions follow from them. On the basis of these influence functions, the integral formula of Green's type can be deduced. 


\section{Generalization of the Green's Integral Formula onto Dynamical Thermoelasticity}

To transform the integral Green's type formula from Cartesian system of coordinates [13] onto cylindrical one, first we need to rewrite the respective influence functions. So, the desired formulae can be rewritten in the cylindrical system of coordinates:

(a) The formula for the influence functions corresponding to a unit point heat flux $a\left[\partial T(\widetilde{M}, \tau) / \partial n_{\widetilde{M}}\right]=\delta(\widetilde{M}-N) \delta(t-\tau)$ on the surface $\Gamma_{N}$ and representing the dynamical thermoelastic displacements

$$
\begin{aligned}
U_{q N}(\tilde{M}, N ; t-\tau)= & \lim _{M \rightarrow \widetilde{M}} U_{q}(M, N ; t-\tau) \\
= & \lim _{M \rightarrow \widetilde{M}} \gamma \int_{0}^{t} d \varsigma \int_{V} G(M, \bar{N} ; t-\varsigma) \Theta^{(q)}(\bar{N}, N ; \varsigma-\tau) d V(\bar{N}) \\
& M, N, \bar{N} \in V ; \quad \widetilde{M} \in \Gamma_{N}
\end{aligned}
$$

(b) The formula for the influence functions corresponding to a unit point temperature $T(\widetilde{M}, r)=-\delta(\widetilde{M}-N) \delta(t-\tau)$ on the surface $\Gamma_{D}$ and representing the dynamical thermoelastic displacements

$$
\begin{aligned}
\frac{\partial U_{q}(\widetilde{M}, N ; t-\tau)}{\partial n_{\tilde{M}}=} & \lim _{M \rightarrow \widetilde{M}} \frac{\partial U_{q}(M, N ; t-\tau)}{\partial n_{M}} \\
= & \gamma \int_{0}^{t} d \varsigma \int_{V} \frac{\partial G(\widetilde{M}, \bar{N} ; t-\varsigma)}{\partial n_{\tilde{M}}} \Theta^{(q)}(\bar{N}, N ; \varsigma-\tau) d V(\bar{N}) \\
& M, N, \bar{N} \in V, \quad \widetilde{M} \in \Gamma_{D}
\end{aligned}
$$

(c) The formula for the influence functions corresponding to an initial unit temperature field $T_{0}(M)=\delta(M-N)$, described by Dirac's delta function $\delta$ and representing the dynamical thermoelastic displacements

$$
\begin{aligned}
U_{q}(M, N ; t) & =\lim _{\tau \rightarrow 0} U_{q}(M, N ; t-\tau) \\
& =\lim _{\tau \rightarrow 0} \gamma \int_{0}^{t} d \varsigma \int_{V} G(M, \bar{N} ; t-\varsigma) \Theta^{(q)}(\bar{N}, N ; \varsigma-\tau) d V(\bar{N})
\end{aligned}
$$

(d) The formula for the influence functions corresponding to a unit point heat exchange of the body with exterior medium $\operatorname{ar}(\widetilde{M}, \tau)+\alpha_{T}\left[\partial T(\widetilde{M}, \tau) / \partial n_{\widetilde{M}}\right]=$ $\delta(\widetilde{M}-N) \delta(t-\tau)$ through the surface $\Gamma_{M}$ and representing the dynamical thermoelastic displacements

$$
\begin{aligned}
U_{q M}(\tilde{M}, N ; t-\tau)= & \lim _{M \rightarrow \widetilde{M}} U_{q}(M, N ; t-\tau) \\
= & \lim _{M \rightarrow \widetilde{M}} \gamma \int_{0}^{t} d \varsigma \int_{V} G(M, \bar{N} ; t-\varsigma) \Theta^{(q)}(\bar{N}, N ; \varsigma-\tau) d V(\bar{N}) \\
& M, N, \bar{N} \in V ; \quad \widetilde{M} \in \Gamma_{M}
\end{aligned}
$$


So, from the definitions of the influence functions in Eqs. (10)-(13) it follow that the function $U_{q}(M, N ; t-\tau)$, defined by Eq. (1), is the main influence function, because other influence functions are obtained from it. The theory of constructing such main influence function in Cartesian coordinates is given in [13]. A number of applications of this theory for particular static and dynamic BVP's in the Cartesian and other systems of coordinates have been presented in [8-17]. In the case of classical uncoupled dynamical thermoelasticity we have to solve the following problems:

(1) The non-stationary heat conduction problem which consists of:

(a) the non-homogeneous non-stationary heat conduction equation [18]

$$
\left(\frac{\partial}{\partial t}-a \nabla_{M}^{2}\right) T(M, t)=F(M, t) ; \quad M \in V, M \equiv(r, \varphi, z)
$$

(b) the non-homogeneous initial condition

$$
T(M, t=0)=T_{0}(M)
$$

written at an inner point $M \in V$, and

(c) non-homogeneous boundary conditions

$$
\begin{aligned}
& \left.T\right|_{\Gamma_{D}}=T(\tilde{M}, t) ; \quad \tilde{M} \in \Gamma_{D} ;\left.\quad a \frac{\partial T}{\partial n_{M}}\right|_{\Gamma_{N}}=a \frac{\partial T(\tilde{M}, t)}{\partial n_{\tilde{M}}} ; \quad \tilde{M} \in \Gamma_{N} \\
& \left.\left(\alpha \frac{\partial}{\partial n_{M}}+a\right) T(M, t)\right|_{\Gamma_{M}}=\left(\alpha \frac{\partial}{\partial n_{\bar{M}}}+a\right) T(\tilde{M}, t) ; \quad \tilde{M} \equiv(\tilde{r}, \tilde{\varphi}, \tilde{z})
\end{aligned}
$$

written at points $\tilde{M}$ of the surface $\Gamma \equiv \Gamma_{D}+\Gamma_{N}+\Gamma_{M}$.

(2) The BVP of dynamical thermoelasticity, which consist of:

(a) the non-homogeneous dynamic equations for the displacements $U_{S}$; $s=$ $r, \varphi, z$

$$
\begin{aligned}
& \mu\left(\nabla^{2} U_{r}-\frac{U_{r}}{r^{2}}-\frac{2}{r^{2}} \frac{\partial U_{\varphi}}{\partial \varphi}\right)+(\lambda+\mu) \frac{\partial \Theta}{\partial r}-\rho_{0} \frac{\partial^{2} U_{r}}{\partial t^{2}}-\gamma \frac{\partial T}{\partial r}=0 \\
& \mu\left(\nabla^{2} U_{\varphi}-\frac{U_{\varphi}}{r^{2}}+\frac{2}{r^{2}} \frac{\partial U_{r}}{\partial \varphi}\right)+(\lambda+\mu) \frac{1}{r} \frac{\partial \Theta}{\partial \varphi}-\rho_{0} \frac{\partial^{2} U_{\varphi}}{\partial t^{2}}-\gamma \frac{1}{r} \frac{\partial T}{\partial \varphi}=0 \\
& \mu \nabla^{2} U_{z}+(\lambda+\mu) \frac{\partial \Theta}{\partial z}-\rho_{0} \frac{\partial^{2} U_{z}}{\partial t^{2}}-\gamma \frac{\partial T}{\partial z}=0
\end{aligned}
$$

(b) the homogeneous initial conditions

$$
U_{S}(M, t=0)=0 ; \quad \frac{\partial U_{S}(M, t=0)}{\partial t}=0
$$

written at an inner point $M \in V$, and 
(c) the homogeneous boundary conditions

$$
\begin{aligned}
& U_{S}(\bar{M}, t)=0 ; \quad \bar{M} \in \Gamma_{U} ; \\
& P_{S}(\bar{M}, t)=0 ; \quad P_{S}=\sigma_{S l} n_{l} ; \quad \bar{M} \in \Gamma_{P} ; \\
& \beta_{1} U_{S}(\bar{M}, t)+\beta_{2} P_{S}(\bar{M}, t)=0 ; \quad \bar{M} \in \Gamma_{U P}
\end{aligned}
$$

written at points $\bar{M}$ of the surface $\Gamma \equiv \Gamma_{U}+\Gamma_{P}+\Gamma_{U P}$. Here the thermoelastic stresses $\sigma_{S l}$ are determined by the Duhamel-Neumann law

$$
\sigma_{S l}=2 \mu \varepsilon_{S l}+\delta_{S l}(\lambda \Theta-\gamma T) ; \quad s, l=r, \varphi, z
$$

Next, using the superposition principle, on the basis of the formulae in Eqs. (1) and (10)-(13), we obtain the following Green's type integral solution of the non-homogeneous BVP of dynamical uncoupled thermoelasticity described by Eqs. (14)-(19):

$$
\begin{aligned}
U_{q}(N, t)= & \int_{V} T_{0}(M) U_{q}(M, N ; t) d V(M)+\int_{0}^{t} d \tau \int_{V} F(M, \tau) U_{q}(M, N ; t-\tau) d V(M) \\
& -a \int_{0}^{t} d \tau \int_{\Gamma_{D}} T(\tilde{M}, \tau) \frac{\partial U_{q}(\tilde{M}, N ; t-\tau)}{\partial n_{\tilde{M}}} d \Gamma_{D}(\tilde{M}) \\
& +a \int_{0}^{t} d \tau \int_{\Gamma_{N}} \frac{\partial T(\tilde{M}, \tau)}{\partial n_{\bar{M}}} U_{q N}(\tilde{M}, N ; t-\tau) d \Gamma_{N}(\tilde{M}) \\
& +\int_{0}^{t} d \tau \int_{\Gamma_{M}}\left[a T(\tilde{M}, \tau)+\alpha \frac{\partial T(\tilde{M}, \tau)}{\partial n_{\tilde{M}}}\right] U_{q M}(\tilde{M}, N ; t-\tau) d \Gamma_{M}(\widetilde{M})
\end{aligned}
$$

Clearly, Eq. (21) represents a generalization of the well-known Green's integral formula of heat conduction theory or of elasticity onto an uncoupled dynamical thermoelasticity in the cylindrical coordinates. Equation (21) can also be trated as a generalization of the classical integral Maysel's formula onto the uncoupled dynamical thermoelasticity. Moreover, Eq. (21) is a generalization of Maysel's formula $[2,4-6]$ for cases when the temperature field satisfies the heat conduction equation corresponding to the heat sources prescribed above. So, we have obtained a new general integral formula of Green's type to determine the displacements in a dynamical uncoupled thermoelasticity written in cylindrical coordinates. The advantage is that it allows us to unite the two-stage process of solving a BVP in the theory of thermal stresses, described by Eqs. (14)-(19), into one single stage described by Eq. (21). The first stage covers determination of a temperature field described by Eqs. (14)-(16). The second stage deals with determination (on the basis of already known temperature) of the thermoelastic displacements, described by Eqs. (17)-(19). As a result, for a particular BVP the new general integral formula (21) may be used to obtain an integral solution of the dynamic uncoupled thermoelasticity. 


\section{VOLUME DILATATION AND FUNDAMENTAL SOLUTIONS FOR A CLASS OF PROBLEMS}

\section{General Integral Representation for Volume Dilatation}

As was mentioned before, to construct the functions $\Theta^{(q)}(M, N ; \varsigma-\tau)$ we need to solve the boundary value problem of dynamical elasticity described by Eqs. (2)-(4). To this end, to construct the influence function corresponding to a unit concentrated body force and representing a volume dilatation $\Theta^{(q)}$, we use the differential equation

$$
\begin{aligned}
& {\left[\rho_{0}(\lambda+2 \mu)^{-1} \frac{\partial^{2}}{\partial t^{2}}-\nabla_{M}^{2}\right] \Theta^{(q)}(M, N ; t-\tau)=(\lambda+2 \mu)^{-1} L^{(S)} \delta(M-N) \delta(t-\tau)} \\
& M, N \in V, M \equiv(r, \varphi, z) ; \quad N \equiv(\rho, \psi, \xi) ; \quad t, \tau \geq 0, t \geq \tau \\
& \nabla_{M}^{2}=\frac{\partial^{2}}{\partial r^{2}}+\frac{1}{r} \frac{\partial}{\partial r}+\frac{1}{r^{2}} \frac{\partial^{2}}{\partial \varphi^{2}}+\frac{\partial^{2}}{\partial z^{2}} \quad L^{(S)}=\delta_{S \rho} \frac{\partial}{\partial r}+\frac{\delta_{S \psi}}{r} \frac{\partial}{\partial \varphi}+\delta_{S \xi} \frac{\partial}{\partial \xi}
\end{aligned}
$$

and the homogeneous initial conditions

$$
\left.\Theta^{(q)}(M, N ; t-\tau)\right|_{t-\tau=0}=0 ;\left.\quad \frac{\partial}{\partial t} \Theta^{(q)}(M, N ; t-\tau)\right|_{t-\tau=0}=0
$$

Clearly, the boundary conditions for the functions $\Theta^{(q)}$ depend on a BVP of the dynamical thermoelasticity and may have the form described by Eq. (4). Also, note that Eq. (22) is obtained from Eq. (2) by differentiation with respect to the coordinates of the point $M \equiv(r, \varphi, z)$ and by summation over the subscript, $s=$ $r, \varphi, z$, using the rule

$$
\Theta^{(q)}=\frac{\partial U_{r}^{(q)}}{\partial r}+\frac{U_{r}^{(q)}}{r}+\frac{1}{r} \frac{\partial U_{\varphi}^{(q)}}{\partial \varphi}+\frac{\partial U_{z}^{(q)}}{\partial z}
$$

The solution to Eq. (22) with initial conditions given by Eq. (23) can be represented in the integral form

$$
\begin{aligned}
\Theta^{(q)}(M, N ; t-\tau)= & -\frac{1}{\lambda+2 \mu} L^{(q)} G_{\Theta}(M, N ; t-\tau) \\
& +\int_{0}^{t} d p \int_{\Gamma}\left[\frac{\partial}{\partial n_{\bar{M}}} \Theta^{(q)}(\bar{M}, N ; p-\tau)-\Theta^{(q)}\left(M^{\prime}, N ; p-\tau\right) \frac{\partial}{\partial n_{\bar{M}}}\right] \\
& \times G_{\Theta}(\bar{M}, M ; t-p) d \Gamma(\bar{M})
\end{aligned}
$$

Here Green's function $G_{\Theta}(M, N ; t-\tau)$ satisfies equation [see Eq. (22)]:

$$
\begin{gathered}
{\left[\rho_{0}(\lambda+2 \mu)^{-1} \frac{\partial^{2}}{\partial t^{2}}-\nabla_{M}^{2}\right] G_{\Theta}(M, N ; t-\tau)=(\lambda+2 \mu)^{-1} \delta(M-N) \delta(t-\tau)} \\
M, N \in V, M \equiv(r, \varphi, z) ; \quad N \equiv(\rho, \psi, \xi) ; \quad t, \tau \geq 0, \quad t \geq \tau
\end{gathered}
$$


subject to the initial conditions [see Eq. (23)]

$$
\left.G_{\Theta}(M, N ; t-\tau)\right|_{t-\tau=0}=0 ;\left.\quad \frac{\partial}{\partial t} G_{\Theta}(M, N ; t-\tau)\right|_{t-\tau=0}=0
$$

Also note that the functions $\Theta^{(q)}$ and $\partial \Theta^{(q)} / \partial n_{\bar{M}}$ on the surface $\Gamma$ depend on the specific boundary conditions similar to those in Eq. (4). In a general case, to obtain those functions, a boundary integral equation is to be solved. However, there is a number of BVP's of the dynamical uncoupled thermoelasticity for which the values of $\Theta^{(q)}$ or $\partial \Theta^{(q)} / \partial n_{\bar{M}}$ are determined directly from the relationship (24), Hooke's law, equilibrium equations and prescribed boundary conditions. In the following, we present an example of determination of the functions $\Theta^{(q)}$ or $\partial \Theta^{(q)} / \partial n_{\bar{M}}$ on the surface $\Gamma$ and of the influence function $\Theta^{(q)}$ for a class of BVP's in the cylindrical coordinates without solving a boundary integral equation.

\section{A Theorem About Volume Dilatation and Fundamental Solutions}

Theorem. Let on the plane surfaces $\Gamma_{z l}$ and $\Gamma_{\varphi n}$ of a cylindrical body $V$, those curvilinear surfaces are placed at infinity, the following mixed boundary conditions are prescribed: 1) normal stresses and tangential displacements, or 2) normal displacements and tangential stresses. Also, let the above conditions be interrelated with the boundary conditions for Green's function $G_{\Theta}$ by

$$
\begin{gathered}
\sigma_{z z}^{(q)}=U_{r}^{(q)}=U_{\varphi}^{(q)}=0 ; \quad G_{\Theta}=0 \\
U_{z}^{(q)}=\sigma_{z r}^{(q)}=\sigma_{z \varphi}^{(q)}=0 ; \quad \partial G_{\Theta} / \partial n_{z l}=0
\end{gathered}
$$

on the surfaces $\Gamma_{z l}\left(0 \leq r<\infty ; 0 \leq \varphi \leq \alpha_{n} ; z=a_{l}\right)$, and

$$
\begin{gathered}
\sigma_{\varphi \varphi}^{(q)}=U_{z}^{(q)}=U_{r}^{(q)}=0 ; \quad G_{\Theta}=0 \\
U_{\varphi}^{(q)}=\sigma_{\varphi z}^{(q)}=\sigma_{\varphi r}^{(q)}=0 ; \quad \partial G_{\Theta} / \partial n_{\varphi n}=0
\end{gathered}
$$

on the surfaces $\Gamma_{\varphi n}\left(0 \leq r<\infty ; \varphi=\alpha_{n} ; 0 \leq z \leq a_{l}\right) ; l, n=0,1$.

Then for the homogeneous initial conditions, as in Eq. (23), the influence functions for volume dilatation $\Theta^{(q)}$ are represented in terms of $G_{\Theta}(M, N ; t-\tau)$ by

$$
\Theta^{(q)}(M, N ; t-\tau)=-\frac{1}{\lambda+2 \mu} L^{(q)} G_{\Theta}(M, N ; t-\tau)
$$

In addition, the influence functions corresponding to an inner unit point heat source and representing the thermoelastic displacements [the fundamental solutions] of uncoupled dynamical thermoelasticity, are determined by:

$$
\begin{aligned}
U_{q}(M, N ; t-\tau) & =-\gamma(\lambda+2 \mu)^{-1} L^{(q)} \int_{0}^{t} d \varsigma \int_{V} G(M, \bar{N} ; t-\varsigma) G_{\Theta}(\bar{N}, N ; t-\tau) d V(\bar{N}) \\
L^{(q)} & =\delta_{q \rho} \frac{\partial}{\partial \rho}+\frac{\delta_{q \psi}}{\rho} \frac{\partial}{\partial \psi}+\delta_{q \xi} \frac{\partial}{\partial \xi}
\end{aligned}
$$


In Eq. (32), the function $G(M, \bar{N} ; t-s)$ is Green's function described by Eqs. (5)-(7), while the function $G_{\Theta}(\bar{N}, N ; t-\tau)$ is Green's function described by Eqs. (26)-(31).

Proof. To obtain Eq. (32) we use the generalized Hooke's law

$$
\sigma_{s p}=2 \mu \varepsilon_{s p}+\delta_{s p} \lambda \Theta ; \quad s, p=r, \varphi, z
$$

and the relationship between strains and displacements

$$
\begin{aligned}
& \varepsilon_{z z}=\frac{\partial U_{z}}{\partial z} ; \quad \varepsilon_{r r}=\frac{\partial U_{r}}{\partial r} ; \quad \varepsilon_{\varphi \varphi}=\frac{1}{r}\left(\frac{\partial U_{\varphi}}{\partial \varphi}+U_{r}\right) ; \quad \varepsilon_{z r}=\frac{1}{2}\left(\frac{\partial U_{z}}{\partial r}+\frac{\partial U_{r}}{\partial z}\right) \\
& \varepsilon_{z \varphi}=\frac{1}{2}\left(\frac{\partial U_{\varphi}}{\partial z}+\frac{\partial U_{z}}{\partial \varphi}\right) ; \quad \varepsilon_{r \varphi}=\frac{1}{2}\left[\frac{1}{r}\left(\frac{\partial U_{r}}{\partial \varphi}-U_{\varphi}\right)+\frac{\partial U_{\varphi}}{\partial r}\right]
\end{aligned}
$$

as well as the formula for the volume dilatation in Eq. (24) written in cylindrical coordinates $(r, \varphi, z)$ for the points of surfaces $\Gamma_{z l}\left(0 \leq r<\infty ; 0 \leq \varphi \leq \alpha_{n} ; z=a_{l}\right)$ and $\Gamma_{\varphi n}\left(0 \leq r<\infty ; \varphi=\alpha_{n} ; 0 \leq z \leq a_{l}\right)$. First, we have to prove that from the mixed boundary conditions given in Eqs. (28) and (30) it follows that on the sides $\Gamma_{z l}\left(0 \leq r<\infty ; 0 \leq \varphi \leq \alpha_{n} ; z=a_{l}\right)$ and $\Gamma_{\varphi n}\left(0 \leq r<\infty ; \varphi=\alpha_{n} ; 0 \leq z \leq a_{l}\right)$ the dilatation vanishes, that is, $\Theta^{(q)}(\bar{M}, N ; t-\tau)=0$. To this end, taking into account the relationship in Eq. (24), written in the form

$$
\partial U_{z}^{(q)} / \partial z=\Theta^{(q)}-\left(\partial U_{r}^{(q)} / \partial r+r^{-1} \partial U_{\varphi}^{(q)} / \partial \varphi+r^{-1} U_{r}^{(q)}\right)
$$

and using Hooke's law, as in Eqs. (34) and (35) written for stresses $\sigma_{z z}^{(q)}$, we obtain

$$
\sigma_{z z}^{(q)}=2 \mu \partial U_{z}^{(q)} / \partial z+\lambda \Theta^{(q)}=(\lambda+2 \mu) \Theta^{(q)}-2 \mu\left(\partial U_{r}^{(q)} / \partial r+r^{-1} \partial U_{\varphi}^{(q)} / \partial \varphi+r^{-1} U_{r}^{(q)}\right)
$$

Also, using in Eq. (37) the conditions $\sigma_{z z}^{(q)}=0 ; U_{r}^{(q)}=0 ; \partial U_{r}^{(q)} / \partial r=0 ; \partial U_{\varphi}^{(q)} / r \partial \varphi=$ 0 , which follow from the boundary conditions Eq. (28), we find that on the sides $\Gamma_{z l}\left(0 \leq r<\infty ; 0 \leq \varphi \leq \alpha_{n} ; z=a_{l}\right)$ the volume dilatation vanishes: $\Theta^{(q)}(\bar{M}, N ; t-\tau)=0, \bar{M} \equiv\left(\bar{r}, \bar{\varphi}, a_{l}\right) \in \Gamma_{1 l}$.

In an analogous way, taking into account the Eq. (24) in the form

$$
r^{-1}\left(\partial U_{\varphi}^{(q)} / \partial \varphi+U_{r}^{(q)}\right)=\Theta^{(q)}-\left(\partial U_{r}^{(q)} / \partial r+\partial U_{z}^{(q)} / \partial z\right)
$$

and using Hooke's law, as in Eqs. (34) and (35), written for stresses $\sigma_{\varphi \varphi}^{(q)}$, we obtain

$$
\sigma_{\varphi \varphi}^{(q)}=2 \mu r^{-1}\left(\partial U_{\varphi}^{(q)} / \partial \varphi+U_{r}^{(q)}\right)+\lambda \Theta^{(q)}=(\lambda+2 \mu) \Theta^{(q)}-2 \mu\left(\partial U_{r}^{(q)} / \partial r+\partial U_{z}^{(q)} / \partial z\right)
$$

Also by using (39) with the conditions $\sigma_{\varphi \varphi}^{(q)}=0 ; \partial U_{r}^{(q)} / \partial r=0 ; \partial U_{z}^{(q)} / \partial z=0$, which follow from the boundary conditions (30), we find that on the sides $\Gamma_{\varphi n}\left(0 \leq r<\infty ; \varphi=\alpha_{n} ; 0 \leq z \leq a_{l}\right)$ the volume dilatation is zero:

$$
\Theta^{(q)}(\bar{M}, N ; t-\tau)=0 ; \quad \bar{M} \equiv\left(, \bar{r}, \alpha_{n}, \bar{z}\right) \in \Gamma_{\varphi n}
$$


In addition, the mixed boundary conditions from Eqs. (29) and (31) imply that the derivatives of the volume dilatation along the external normal on the sides $\Gamma_{z l}$ and $\Gamma_{\varphi n}$ vanish, that is, on these sides $\partial \Theta^{(q)} / \partial n_{z l}=0$ and $\partial \Theta^{(q)} / \partial n_{z l}=0$. For example, for the sides $\Gamma_{z l}\left(0 \leq r<\infty ; 0 \leq \varphi \leq \alpha_{n} ; z=a_{l}\right)$, by substituting the relations

$$
\partial \sigma_{z r}^{(q)} / \partial r=0 ; \quad r^{-1} \sigma_{z r}^{(q)}=0 ; \quad \sigma_{z \varphi}^{(q)}=0 ; \quad r^{-1}\left(\partial \sigma_{z \varphi}^{(q)} / \partial \varphi\right)=0
$$

which follow from the boundary conditions in Eq. (29), into the stresses equilibrium equation

$$
\frac{\partial \sigma_{z r}^{(q)}}{\partial r}+\frac{\sigma_{z r}^{(q)}}{r}+\frac{\partial \sigma_{z \varphi}^{(q)}}{r \partial \varphi}+\frac{\partial \sigma_{z z}^{(q)}}{\partial z}=0
$$

we obtain that on this side $\partial \sigma_{z z}^{(q)} / \partial z=0$. So, from Eq. (37) and the last result, we obtain

$$
\frac{\partial \sigma_{z z}^{(q)}}{\partial z}=-\frac{\partial \sigma_{z z}^{(q)}}{\partial n_{z l}}=(\lambda+2 \mu) \frac{\partial \Theta^{(q)}}{\partial z}-2 \mu\left[\frac{\partial^{2} U_{r}^{(q)}}{\partial r \partial z}+\frac{1}{r}\left(\frac{\partial^{2} U_{\varphi}^{(q)}}{\partial \varphi \partial z}+\frac{\partial U_{r}^{(q)}}{\partial z}\right)\right]=0
$$

Next, using the boundary conditions from Eq. (29) and Hooke's law for the tangential stresses $\sigma_{z r}^{(q)}$ and $\sigma_{z \varphi}^{(q)}$, it can be shown that on the sides $\Gamma_{z l}\left(0 \leq r<\infty ; 0 \leq \varphi \leq \alpha_{n} ; z=a_{l}\right)$ the following results hold true

$$
\frac{\partial U_{r}^{(q)}}{\partial z}=0 ; \quad \frac{\partial^{2} U_{r}^{(q)}}{\partial r \partial z}=0 ; \quad \frac{\partial^{2} U_{\varphi}^{(q)}}{\partial \varphi \partial z}=0
$$

The result (44) can be proved by using the following relations:

$$
\begin{aligned}
U_{z}^{(q)}=0 & \Rightarrow \frac{\partial U_{z}^{(q)}}{\partial r}=0, \quad \frac{\partial U_{z}^{(q)}}{\partial \varphi}=0 \\
\sigma_{z r}^{(q)}=0, \quad \frac{\partial U_{z}^{(q)}}{\partial r}=0 & \Rightarrow \mu\left(\frac{\partial U_{z}^{(q)}}{\partial r}+\frac{\partial U_{r}^{(q)}}{\partial z}\right)=0 ; \\
& \Rightarrow \frac{\partial U_{r}^{(q)}}{\partial z}=0, \quad \frac{\partial^{2} U_{r}^{(q)}}{\partial r \partial z}=0 \\
\sigma_{z \varphi}^{(q)}=0, \quad \frac{\partial U_{z}^{(q)}}{\partial \varphi}=0 & \Rightarrow \mu\left(\frac{\partial U_{z}^{(q)}}{\partial \varphi}+\frac{\partial U_{\varphi}^{(q)}}{\partial z}\right)=0 ; \\
& \Rightarrow \frac{\partial U_{\varphi}^{(q)}}{\partial z}=0, \quad \frac{\partial^{2} U_{\varphi}^{(q)}}{\partial \varphi \partial z}=0
\end{aligned}
$$

Finally, by combining (44) with (43) and by using the boundary conditions (29), we find that on the sides $\Gamma_{z l}\left(0 \leq r<\infty ; 0 \leq \varphi \leq \alpha_{n} ; z=a_{l}\right)$ there is $\partial \Theta^{(q)} / \partial z=$ 0 or $\partial \Theta^{(q)} / \partial n_{z l}=0$.

In an analogous way, we prove that the boundary conditions (31) imply that on the sides $\Gamma_{\varphi n}\left(0 \leq r<\infty ; \varphi=\alpha_{n} ; 0 \leq z \leq a_{l}\right)$ lead to the following equality $\partial \Theta^{(q)} / \partial n_{\varphi n}=0$. Having substituted the relationships $\sigma_{\varphi r}^{(q)}=0 ; \partial \sigma_{\varphi r}^{(q)} / \partial r=$ 
$0 ; \partial \sigma_{\varphi z}^{(q)} / \partial z=0$, which follow from the boundary conditions in Eq. (31), into the equation of equilibrium in stresses

$$
\partial \sigma_{\varphi r}^{(q)} / \partial r+\partial \sigma_{\varphi \varphi}^{(q)} / r \partial \varphi+\partial \sigma_{\varphi z}^{(q)} / \partial z+2 r^{-1} \sigma_{\varphi r}^{(q)}=0
$$

we obtain that on the sides $\Gamma_{\varphi n}\left(0 \leq r<\infty ; \varphi=\alpha_{n} ; 0 \leq z \leq a_{l}\right)$, the relation $\partial \sigma_{\varphi \varphi}^{(q)} / \partial \varphi=0$ holds. Using this result in Hooke's law (39) in the form

$$
\frac{\partial \sigma_{\varphi \varphi}^{(q)}}{\partial \varphi}=(\lambda+2 \mu) \frac{\partial \Theta^{(q)}}{\partial \varphi}-2 \mu\left(\frac{\partial^{2} U_{r}^{(q)}}{\partial r \partial \varphi}+\frac{\partial^{2} U_{z}^{(q)}}{\partial z \partial \varphi}\right)=0
$$

and the boundary conditions (31), as well as Hooke's law for the tangential stresses $\sigma_{\varphi r}^{(q)}$ and $\sigma_{\varphi z}^{(q)}$, we find that on the sides $\Gamma_{\varphi n}\left(0 \leq r<\infty ; \varphi=\alpha_{n} ; 0 \leq z \leq a_{l}\right)$ the following relations hold:

$$
\partial^{2} U_{r}^{(q)} / \partial r \partial \varphi=0 ; \quad \partial^{2} U_{z}^{(q)} / \partial z \partial \varphi=0
$$

Equation (48) can be obtained by the following operations:

$$
\begin{aligned}
U_{\varphi}^{(q)}=0 & \Rightarrow \frac{\partial U_{\varphi}^{(q)}}{\partial r}=0, \quad \frac{\partial U_{\varphi}^{(q)}}{\partial z}=0 \\
\sigma_{\varphi r}^{(q)}=0, \quad U_{\varphi}^{(q)}=0, \quad \frac{\partial U_{\varphi}^{(q)}}{\partial r}=0 & \Rightarrow \mu\left[\frac{1}{r}\left(\frac{\partial U_{r}^{(q)}}{\partial \varphi}-U_{\varphi}^{(q)}\right)+\frac{\partial U_{\varphi}^{(q)}}{\partial r}\right]=0 \\
& \Rightarrow \frac{\partial U_{r}^{(q)}}{\partial \varphi}=0, \quad \frac{\partial^{2} U_{r}^{(q)}}{\partial r \partial \varphi}=0 \\
\sigma_{\varphi z}^{(q)}=0, \quad \frac{\partial U_{\varphi}^{(q)}}{\partial z}=0 & \Rightarrow \mu\left(\frac{\partial U_{z}^{(q)}}{\partial \varphi}+\frac{\partial U_{\varphi}^{(q)}}{\partial z}\right)=0 \\
& \Rightarrow \frac{\partial U_{z}^{(q)}}{\partial \varphi}=0, \quad \frac{\partial^{2} U_{z}^{(q)}}{\partial \varphi \partial z}=0
\end{aligned}
$$

Finally, by substituting the results (48) into (47) we find that on the sides $\Gamma_{\varphi n}\left(0 \leq r<\infty ; \varphi=\alpha_{n} ; 0 \leq z \leq a_{l}\right)$ there is $\partial \Theta^{(q)} / \partial \varphi=0$ or $\partial \Theta^{(q)} / \partial n_{\varphi n}=0$.

As a result we come to the following conclusions:

i. If on the boundary of a cylindrical body $V+\Gamma$ the normal zero stresses as well as tangential displacements vanish (the boundary conditions from Eqs. (28) and (30)), then on this side $\Theta^{(q)}=G_{\Theta}=0$.

ii. If on a boundary of the cylindrical body $V+\Gamma$ the normal displacements as well as tangential stresses vanish (the boundary conditions from Eqs. (28) and (30)), then on this boundary $\partial \Theta^{(q)} / \partial n=\partial G_{\Theta} / \partial n=0$.

Finally, taking into account the definition of the boundary $\Gamma \equiv \sum_{l=0}^{1} \Gamma_{z l}+\sum_{n=0}^{\alpha}+\Gamma_{\varphi n}$, as well as conclusions $\mathrm{i}$ and ii, we find that Eq. (32) for volume dilatation holds true.

By introducing (32) into (1) we obtain the desired formula (33) for the fundamental solutions $U_{q}(M, N ; t-\tau)$ (the influence functions corresponding to an inner unit point heat source and representing thermoelastic displacements) 
of a wide class of BVP's of dynamical uncoupled thermoelasticity. Clearly, the functions $U_{q}(M, N ; t-\tau)$ are the main influence functions, because other influence functions can be obtained from it. The class of problems includes BVP's of dynamical uncoupled thermoelasticity for the domains described in the cylindrical and polar coordinates whose surfaces are the coordinate planes with equations $\Gamma_{z l}\left(0 \leq r<\infty ; 0 \leq \varphi \leq \alpha_{n} ; z=a_{l}\right)$ and $/$ or $\quad \Gamma_{\varphi n}\left(0 \leq r<\infty ; \varphi=\alpha_{n} ; 0 \leq z \leq a_{l}\right) \quad$ in cylindrical system of coordinates, and coordinate lines $\Gamma_{\varphi n}\left(0 \leq r<\infty ; \varphi=c_{n}\right)$-in the polar system of coordinates. The curvilinear surfaces (boundaries) of such kind of cylindrical (polar) domain are placed at infinity. As examples of these domains the following could serve: (1) 3-D BVP for: quarter of space, eighth part of space or octant, layer, semi-layer, quarter of layer, various wedges, semi-wedges and limited wedges with the different angles $\alpha$, including $0<\alpha=\pi / n ; n=2,3,4, \ldots$,; (2) 2-D BVP for half-plane, quarter of plane (quadrant) and also for different plane wedges, that is, for the canonical polar domains described by equations $0 \leq \varphi \leq \alpha ; 0<\alpha \leq 2 \pi ; 0 \leq r<\infty$.

\section{EXAMPLE OF THE INFLUENCE FUNCTIONS AND GREEN'S TYPE INTEGRAL FORMULA IN DYNAMICAL UNCOUPLED THERMOELASTICITY FOR AN INFINITE WEDGE}

In this section, a general integral formula (21) is to be specified for a particular initial boundary value problem of uncoupled dynamic thermoelasticity for an infinite wedge.

The infinite wedge is described by: $V(0 \leq r<\infty, 0 \leq \varphi \leq \alpha,-\infty<z<\infty), \alpha=$ $(\pi / n) ; n=2,3,4, \ldots$, with the sides $\Gamma_{\varphi 0}(0 \leq \bar{r}<\infty, \bar{\varphi}=0,-\infty<\bar{z}<\infty)$ and $\Gamma_{\varphi \alpha}(0 \leq \bar{r}<\infty, \bar{\varphi}=\alpha,-\infty<\bar{z}<\infty)$; and a dynamical thermoelastic process in the wedge corresponds to an internal heat source $F(M, t), M \equiv(r, \varphi, z) ; M \in V$, a temperature $T(\bar{r}, \bar{\varphi}=0, \bar{z}), \bar{M} \equiv(\bar{r}, \bar{\varphi}=0, \bar{z}) \in \Gamma_{\varphi 0}$ on the side $\Gamma_{\varphi 0}$ and by the heat flux $a\left[\partial T(\bar{r}, \bar{\varphi}=\alpha, \bar{z}) / \partial n_{\alpha}\right], \bar{M} \equiv(\bar{r}, \bar{\varphi}=\alpha, \bar{z}) \in \Gamma_{\varphi \alpha}$ on the side $\Gamma_{\varphi \alpha}$. On the boundary planes $\Gamma_{\varphi 0}$ and $\Gamma_{\varphi \alpha}$ of wedge $V$, the following homogeneous mechanical boundary conditions of the type of sliding and non-homogeneous thermal boundary conditions are postulated

$$
\sigma_{\varphi \varphi}^{(q)}=U_{z}^{(q)}=U_{r}^{(q)}=0 ; \quad T=T_{1} \neq 0
$$

on the side $\Gamma_{\varphi 0}-(\bar{\varphi}=0)$, and

$$
U_{\varphi}^{(q)}=\sigma_{\varphi z}^{(q)}=\sigma_{\varphi r}^{(q)}=0 ; \quad a\left[\partial T / \partial n_{\alpha}\right]=a\left[\partial T_{2} / \partial n_{\alpha}\right] \neq 0
$$

on the side $\Gamma_{\varphi \alpha}-(\bar{\varphi}=\alpha)$.

The mechanical initial conditions for the problem are homogeneous as in (18), while the thermal initial conditions are non-homogeneous as in (15): $T_{0}(M) \neq 0$. To solve this problem in accordance with a traditional scheme it is necessary to solve the heat conduction equation (14) subject to the initial conditions (15) and the non-homogeneous boundary conditions (50) and (51). Next, with temperature $T$ already found, it is necessary to solve the non-homogeneous equations of dynamical thermoelasticity (17) subject to the homogeneous initial conditions (18) and the 
boundary conditions (50)-(51). Clearly, a solution to the problem is represented by the integral formula

$$
\begin{aligned}
U_{q}(N, t)= & \int_{V} T_{0}(M) U_{q}(M, N ; t) d V(M)+\int_{0}^{t} d \tau \int_{V} F(M, \tau) U_{q}(M, N ; t-\tau) d V(M) \\
& -a \int_{0}^{t} d \tau \int_{\Gamma_{\varphi 0}} T_{1}(\bar{M}, \tau) \frac{\partial U_{q}(\bar{M}, N ; t-\tau)}{\partial n_{\bar{M}}} d \Gamma_{\varphi 0}(\bar{M}) \\
& +a \int_{0}^{t} d \tau \int_{\Gamma_{\varphi x}} \frac{\partial T_{2}(\bar{M}, \tau)}{\partial n_{\bar{M}}} U_{q N}(\bar{M}, N ; t-\tau) d \Gamma_{\varphi x}(\bar{M})
\end{aligned}
$$

The formula (52) is obtained from (21), in which $\Gamma_{D} \equiv \Gamma_{\varphi 0}, \Gamma_{N} \equiv \Gamma_{\varphi \alpha}, \Gamma_{M} \equiv$ $0, T_{0} \equiv 0$. In Eq. (52) the influence function $U_{q}(M, N ; t-\tau)$ is determined by the integral formula (1).

To obtain $U_{q}(M, N ; t-\tau)$, Green's function $G(M, N ; t-\tau)$ should be constructed first by solving the following initial boundary value heat conduction problem for the wedge $V$ with the boundaries $\Gamma_{\varphi 0}$ and $\Gamma_{\varphi \alpha}$

$$
\begin{aligned}
& \left(\frac{\partial}{\partial t}-a \nabla_{M}^{2}\right) G(M, N ; t-\tau)=\delta(M-N) \delta(t-\tau) ; \\
& \quad M, N \in V, M \equiv(r, \varphi, z) ; \quad N \equiv(\rho, \psi, \xi) ; \quad t, \tau \geq 0, t>\tau
\end{aligned}
$$

subject to the homogeneous initial and boundary conditions on the sides $\Gamma_{\varphi 0}$ and $\Gamma_{\varphi \alpha}$

$$
\begin{gathered}
\left.G(M, N ; t-\tau)\right|_{t-\tau=0}=0 ; \quad G(\bar{M}, N ; t-\tau)=0 ; \quad \bar{M} \in \Gamma_{\varphi 0} ; \\
\partial G(\bar{M}, N ; t-\tau) / \partial n_{\alpha}=0 ; \quad \bar{M} \in \Gamma_{\varphi \alpha}
\end{gathered}
$$

This is a particular case of the general BVP described by Eqs. (5)-(7).

A solution to the BVP (53)-(54), that is, the desired Green's function $G$, obtained by the method of reflection [19], takes the form:

$$
\begin{aligned}
G(M, N ; t-\tau) & =\left[8 \sqrt{\pi^{3} a^{3}(t-\tau)^{3}}\right]^{-1} \sum_{k=0}^{n-1}(-1)^{(k)}\left(e^{-\frac{R_{k}^{2}}{4 a(t-\tau)}}-e^{-\frac{R_{k \psi}^{2}}{4 a(t-\tau)}}\right) \\
R_{k} & =\sqrt{r^{2}+\rho^{2}-2 r \rho \cos \left(\varphi-\psi-\frac{2 k \pi}{n}\right)+(z-\xi)^{2}} ; \\
R_{k \psi} & =\sqrt{r^{2}+\rho^{2}-2 r \rho \cos \left(\varphi+\psi-\frac{2 k \pi}{n}\right)+(z-\xi)^{2}}
\end{aligned}
$$

Next, to construct the influence functions $\Theta^{(q)}$, we are to solve Eqs. (2) subject to the homogeneous initial conditions (3) and non-homogeneous boundary conditions (50)-(51). Using the theorem on dilatation, we conclude that the functions $\Theta^{(q)}$ satisfying the BVP, described by Eqs. (2), (3), and (50)-(51), are 
determined by Eq. (32), wherein the influence function $G_{\Theta}$ satisfies Eq. (26) subject to the following homogeneous initial and boundary conditions:

$$
\begin{gathered}
\left.G_{\Theta}(M, N ; t-\tau)\right|_{t-\tau=0}=0 ;\left.\quad \frac{\partial G_{\Theta}(M, N ; t-\tau)}{\partial t}\right|_{t-\tau=0}=0 \\
G_{\Theta}(\bar{M}, N ; t-\tau)=0 ; \quad \bar{M} \in \Gamma_{\varphi 0} ; \quad \partial G_{\Theta}(\bar{M}, N ; t-\tau) / \partial n_{\alpha}=0 ; \quad \bar{M} \in \Gamma_{\varphi \alpha}
\end{gathered}
$$

Equation (56) follows from a comparison of the homogeneous initial conditions (18) and boundary conditions (50)-(51) with the corresponding conditions (23) and (30)(31) of the theorem on volume dilatation.

Finding the influence function $G_{\Theta}$ for the BVP, described by Eqs. (26) and (56), by the method of reflection, and substituting $G_{\Theta}$ into Eq. (21), we obtain the function $\Theta^{(q)}$ in the form:

$$
\begin{aligned}
\Theta^{(q)}(M, N ; t-\tau)= & -\frac{1}{4 \pi(\lambda+2 \mu)} L^{(q)} \sum_{k=0}^{n-1}(-1)^{k} \\
& \times\left[\delta\left(\frac{R_{k}}{c}-(t-\tau)\right) R_{k}^{-1}-\delta\left(\frac{R_{k \psi}}{c}-(t-\tau)\right) R_{k \psi}^{-1}\right] ; \\
c= & \sqrt{\rho_{0}^{-1}(\lambda+2 \mu)}
\end{aligned}
$$

where $c$ is the velocity of propagation of longitudinal waves in an elastic medium.

Finally, if Eq. (57) is substituted into Eq. (1) and the volume integral is calculated, then the influence functions corresponding to an internal unit point heat source $F=\delta(M-N) \delta(t-\tau)$ and representing the dynamical thermoelastic displacements, are obtained in the form

$$
\begin{aligned}
U_{q}(M, N ; t-\tau) & =\gamma \int_{0}^{t} d \varsigma \int_{-\infty}^{\infty} d \bar{z} \int_{0}^{\infty} d \bar{r} \int_{0}^{\alpha} G(M, \bar{N} ; t-\varsigma) \Theta^{(q)}(\bar{N}, N ; \varsigma-\tau) d \bar{\varphi} \\
& =\gamma L^{(q)} \sum_{k=0}^{n-1}(-1)^{k}\left(\Phi_{k}-\Phi_{k \psi}\right) ; \quad \bar{N} \equiv(\bar{r}, \bar{\varphi}, \bar{z}) \in V
\end{aligned}
$$

Here the functions $\Phi_{k}$ are determined by the formula

$$
\begin{aligned}
\Phi_{k}= & \Phi_{k}(M, N ; t-\tau) \\
= & \frac{m}{4 \pi R_{k}}\left\{\left(e^{\left(t^{\bullet}-\tau^{\bullet}\right)-r_{k}}-1\right) H_{k} \cdot\left(\left(t^{\bullet}-\tau^{\bullet}\right)-r_{k}\right)\right. \\
& \left.-\left[U_{k}\left(r_{k},\left(t^{\bullet}-\tau^{\bullet}\right)\right)-\operatorname{erfc}\left(\frac{r_{k}}{2 \sqrt{t^{\bullet}-\tau^{\bullet}}}\right)\right]\right\}
\end{aligned}
$$

in which the first and second terms represent elastic and diffusion fields, respectively, and the functions $U_{k}(r, t-\tau)$ are defined by:

$$
U_{k}(r, t-\tau)=\frac{e^{\boldsymbol{\iota}^{\bullet}-\tau^{\bullet}}}{2}\left[e^{r_{k}} \operatorname{erfc}\left(\frac{r_{k}}{2 \sqrt{t^{\bullet}-\tau^{\bullet}}}+\sqrt{t^{\bullet}-\tau^{\bullet}}\right)\right.
$$




$$
\begin{aligned}
& \left.\quad+e^{-r_{k}} \operatorname{erfc}\left(\frac{r_{k}}{2 \sqrt{t^{\bullet}-\tau^{\bullet}}}-\sqrt{t^{\bullet}-\tau^{\bullet}}\right)\right] \\
& r_{k}=\left(a^{-1} c\right) R_{k} ; \quad t^{\bullet}-\tau^{\bullet}=\left(a^{-1} c^{2}\right)(t-\tau) ; \quad m=\gamma c^{-2} \rho_{0}^{-1}
\end{aligned}
$$

In Eq. (59), the Heaviside function $H$ is defined by the formula

$$
H_{k} \equiv H_{k}\left(\left(t^{\bullet}-\tau^{\bullet}\right)-r_{k}\right) \equiv\left\{\begin{array}{ll}
0 & \left(t^{\bullet}-\tau^{\bullet}\right) \leq r_{k} \text { or }(t-\tau) \leq R_{k} / c \\
1 & \left(t^{\bullet}-\tau^{\bullet}\right)>r_{k} \text { or }(t-\tau)>R_{k} / c
\end{array}\right\}
$$

The functions $\Phi_{k \psi}, k=0,1, \ldots, n-1 ; n=2,3,4, \ldots$, in Eq. (58) are obtained from the functions $\Phi_{k}$ by replacing $\psi$ by $\psi^{\bullet}=-\psi$.

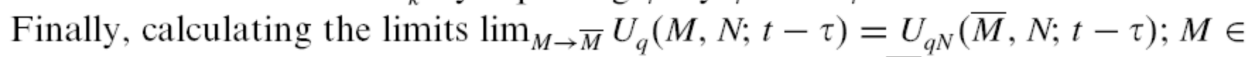
$V ; \bar{M} \in \Gamma_{\varphi \alpha} \quad$ and $\quad \lim _{M \rightarrow \bar{M}} \partial U_{q}(M, N ; t-\tau) / \partial n_{\varphi 0}=\partial U_{q s}(\bar{M}, N ; t-\tau) / \partial n_{\varphi 0} ; M \in$ $V, \bar{M} \in \Gamma_{\varphi 0}$; and using (52), the following integral formula of Green's type for the thermoelastic dynamical displacements is obtained

$$
\begin{aligned}
U_{q}(\rho, \psi, \xi, t)= & \gamma L^{(q)} \sum_{k=0}^{n-1}(-1)^{k}\left\{\int_{-\infty}^{\infty} d z \int_{0}^{\infty} r d r \int_{0}^{\alpha} T_{0}(r, \varphi, z)\right. \\
& \times\left(\Phi_{k}(r, \varphi, z ; \xi, \rho, \psi ; t)-\Phi_{k \psi}(r, \varphi, z ; \xi, \rho, \psi ; t)\right) d \varphi \\
& +\int_{0}^{t} d \tau \int_{-\infty}^{\infty} d z \int_{0}^{\infty} r d r \int_{0}^{\alpha} F(r, \varphi, z ; \tau) \\
& \times\left(\Phi_{k}(r, \varphi, z ; \xi, \rho, \psi ; t-\tau)-\Phi_{k \psi}(r, \varphi, z ; \xi, \rho, \psi ; t-\tau)\right) d \varphi \\
& -a \int_{0}^{t} d \tau \int_{-\infty}^{\infty} d \bar{z} \int_{0}^{\infty} T_{1}(\bar{r}, \varphi=0, \bar{z} ; \tau) \\
& \times \frac{\partial}{\partial n_{\varphi 0}}\left[\Phi_{k}(\bar{r}, \varphi=0, \bar{z} ; \xi, \rho, \psi ; t-\tau)\right. \\
& \left.-\Phi_{k \psi}(\bar{r}, \varphi=0, \bar{z} ; \xi, \rho, \psi ; t-\tau)\right] d \bar{r} \\
& +a \int_{0}^{t} d \tau \int_{-\infty}^{\infty} d \bar{z} \int_{0}^{\infty} \frac{\partial T_{2}(\bar{r}, \varphi=\alpha, \bar{z} ; \tau)}{\partial n_{\varphi \alpha}} \\
& \left.\times\left[\Phi_{k}(\bar{r}, \varphi=\alpha, \bar{z} ; \xi, \rho, \psi ; t-\tau)-\Phi_{k \psi}(\bar{r}, \varphi=\alpha, \bar{z} ; \xi, \rho, \psi ; t-\tau)\right] d \bar{r}\right\}
\end{aligned}
$$

The functions given by Eq. (62) satisfies to equations

$$
\begin{gathered}
\mu\left(\nabla^{2} U_{r}-\frac{U_{r}}{r^{2}}-\frac{2}{r^{2}} \frac{\partial U_{\varphi}}{\partial \varphi}\right)+(\lambda+\mu) \frac{\partial \Theta}{\partial r}-\rho_{0} \frac{\partial^{2} U_{r}}{\partial t^{2}}-\gamma \frac{\partial T}{\partial r}=0 \\
\mu\left(\nabla^{2} U_{\varphi}-\frac{U_{\varphi}}{r^{2}}+\frac{2}{r^{2}} \frac{\partial U_{r}}{\partial \varphi}\right)+(\lambda+\mu) \frac{1}{r} \frac{\partial \Theta}{\partial \varphi}-\rho_{0} \frac{\partial^{2} U_{\varphi}}{\partial t^{2}}-\gamma \frac{1}{r} \frac{\partial T}{\partial \varphi}=0 \\
\mu \nabla^{2} U_{z}+(\lambda+\mu) \frac{\partial \Theta}{\partial z}-\rho_{0} \frac{\partial^{2} U_{z}}{\partial t^{2}}-\gamma \frac{\partial T}{\partial z}=0
\end{gathered}
$$


subject to the homogeneous initial conditions in Eq. (18) and the non-homogeneous boundary conditions of the type described in Eqs. (50) and (51).

Note that the integrals over infinite intervals (62) exist if the thermal data comply with the conditions:

$$
\begin{gathered}
\int_{-\infty}^{\infty} d z \int_{0}^{\infty} r d r \int_{0}^{\alpha}\left|T_{0}(r, \varphi, z)\right| d \varphi<\infty ; \int_{-\infty}^{\infty} d z \int_{0}^{\infty}|F(r, \varphi, z ; \tau)| r d r<\infty ; \\
\int_{-\infty}^{\infty} d \bar{z} \int_{0}^{\infty}\left|T_{1}(\bar{r}, \varphi=0, \bar{z} ; \tau)\right| d \bar{r}<\infty ; \int_{-\infty}^{\infty} d \bar{z} \int_{0}^{\infty}\left|\frac{\partial T_{2}(\bar{r}, \varphi=\alpha, \bar{z} ; \tau)}{\partial n_{\varphi \alpha}}\right| d \bar{r}<\infty
\end{gathered}
$$

It can be proved that the conditions (64) are satisfied if:

1. The thermal data are prescribed on the bounded domains; and

2. The thermal data vanish at infinity.

In both these cases the displacements $U_{q}(\rho, \psi, \xi, t)$ defined by (62) are finite, that is, $\left|U_{q}(\rho, \psi, \xi, t)\right|<\infty$, and because the kernels in (62) vanish at infinity, the functions $U_{q}(\rho, \psi, \xi, t)$ also vanish at infinity.

The authors believe that the solution to dynamic uncoupled thermoelasticity, described by Eqs. (58)-(62) has been obtained for the first time in the literature. In the particular case when $\alpha=\pi / 2$, the wedge is transformed to a quarter of space and the formula (62) is transformed to the integral formula for thermoelastic dynamical displacements for a quarter of space obtained in Cartesian coordinates [13].

Also, influence function formulae, as well as the general integral formula obtained in the present paper, constitute a generalization of Green's and Mayzel's formulae to include an uncoupled dynamic thermoelasticity in the cylindrical and polar coordinates. The generalized formulae can be used to solve a number of BVP's of the uncoupled dynamical thermoelasticity in terms of elementary functions for canonical domains in polar and cylindrical coordinates, such as: an infinite plane, an infinite 3D space, a half and a quarter of plane, a half and a quarter of the 3D space, an eighth of a 3D space, a 3D wedge and a semi-infinite wedge, and so on. The solutions of the particular BVP's play an important role in the numerical analysis of general initial boundary value problems of uncoupled thermoelasticity. Also, the method of solving the particular initial boundary value problems of uncoupled thermoelasticity for canonical domains in polar and cylindrical coordinates proposed in this paper can be extended to include the domains described in other orthogonal coordinate systems, such as the spherical. Note that the crucial problem of this extension is to prove new theorems on dilatation. This could be done for wide classes of the BVP for particular semi-infinite domains of each orthogonal system of coordinates. Partially, this possibility follows from the results obtained in $[8,12,13,20-22]$.

\section{NOMENCLATURE}

$\alpha_{t}-$ coefficient of the linear thermal expansion

$\lambda, \mu$ - Lame's constants of elasticity

$\gamma=\alpha_{t}(2 \mu+3 \lambda)-$ thermoelastic constant

$\rho_{0}-$ density of the body 
$\alpha$ - coefficient of convective heat conductivity

$a$ - coefficient of temperature conductivity

$c$ - velocity of propagation of longitudinal waves in an elastic medium

$\delta_{q l}-$ Kronecker's symbol

$\tau$ - time of application of the source

$t$ - time of observation

$V$ - body volume

$\Gamma$ - surface of the body $V$

$(r, \varphi, z)$ and $(r, \varphi)$ - cylindrical and polar coordinates

$M(r, \varphi, z) M \in V$ - inner point of the body $V$

$F(M, t)$ - inner heat source

$T(M, t)$ - inner temperature

$T_{0}(M)$ - initial inner temperature

$U_{S}(M, t)$ - inner displacements

$\varepsilon_{s p} ; s, p=r, \varphi, z-$ strains

$\Theta$ - volume dilatation, $\Theta=\partial U_{r} / \partial r+U_{r} / r+\partial U_{\varphi} / r \partial \varphi+\partial U_{z} / \partial z$

$\sigma_{s p}=2 \mu \varepsilon_{s p}+\delta_{s p} \lambda \Theta ; s, p=r, \varphi, z-$ Hooke's law for elastic stresses

$\sigma_{s l}=2 \mu \varepsilon_{s l}+\delta_{s l}(\lambda \Theta-\gamma T) ; s, l=r, \varphi, z$ - Duhamel-Neumann law for elastic thermal stresses

$\bar{M} \equiv(\bar{r}, \bar{\varphi}, \bar{z})-$ point of surface $\Gamma \equiv \Gamma_{U}+\Gamma_{P}+\Gamma_{U P}$

$\Gamma_{U}, \Gamma_{P}$ and $\Gamma_{U P}-$ the surfaces on which the boundary conditions for displacements, tractions, or mixed are prescribe

$U_{S}(\bar{M}, t) ; \bar{M} \in \Gamma_{U}$ - displacements prescribed on the surface $\Gamma_{U}$

$P_{S}(\bar{M}, t)=0 ; P_{S}=\sigma_{S l} n_{l} ; \bar{M} \in \Gamma_{P}$ - tractions prescribed on the surface $\Gamma_{P}$

$n_{l}$ - are the directory cosines on the surface $\Gamma$

$\beta_{1} U_{S}(\bar{M}, t)+\beta_{2} P_{S}(\bar{M}, t)=0 ; \bar{M} \in \Gamma_{U P}-$ linear combination of the displacements and tractions prescribed on the surface $\Gamma_{U P}$

$\beta_{1}$ and $\beta_{2}$ - constants that depend on the mechanical characteristics of the contacting bodies

$\widetilde{M} \equiv(\tilde{r}, \tilde{\varphi}, \tilde{z})-$ point of the surface $\Gamma \equiv \Gamma_{D}+\Gamma_{N}+\Gamma_{M}$

$\Gamma_{D}, \Gamma_{N}$ and $\Gamma_{M}$ - the surfaces on which the boundary conditions of Dirichlet's, Neuman's, or mixed type are prescribed

$T(\widetilde{M}, \tau) ; \tilde{M} \in \Gamma_{D}$ - temperature prescribed on the surface $\Gamma_{D}$

$a\left[\partial T(\widetilde{M}, \tau) / \partial n_{\tilde{M}}\right] \tilde{M} \in \Gamma_{N}$ - heat flux prescribed on the surface $\Gamma_{N}$

$\left(\alpha\left(\partial / \partial n_{\tilde{M}}\right)+a\right) T(\widetilde{M}, t) ; \widetilde{M} \in \Gamma_{M}$ - law of the heat exchange of the body with exterior medium prescribed on the surface $\Gamma_{M}$

$N(\rho, \psi, \xi) N \in V$ - an inner point of application of the unit point source (heat source, body force etc)

$(\rho, \psi, \xi)$ and $(\rho, \psi)$ - cylindrical and polar coordinates of the point of application of the source

$\delta(M-N)$ and $\delta(M-N) \delta(t-\tau)-$ Dirac's delta functions

$G(M, N ; t-\tau) ; t, \tau \geq 0 ; t \geq \tau$ - Green's function for an initial-boundary value heat conduction problem

$\Theta^{(q)}(M, N ; t-\tau) ; t, \tau \geq 0 ; t \geq \tau$ - influence function represents a volume dilatation in an inner point $M$ of elastodynamics problem corresponding to a unit concentrated body force, applied in an inner point $N$ in the direction of the axix $(q \equiv \rho, \psi, \xi)$ 
$G_{\Theta}(M, N ; t-\tau) ; t, \tau \geq 0 ; t \geq \tau-$ Green's function in an inner point $M$ for an initial-boundary value elastodynamics problem for dilatation

$U_{S}^{(q)}(M, N ; t-\tau) ; t, \tau \geq 0 ; t \geq \tau-$ displacements in an inner point of observation $M$ in the direction of the axis $(s \equiv r, \varphi, z)$ corresponding to an inner unit point body force applied in an inner point $N$ in the direction of the axis $(q \equiv \rho, \psi, \xi)$ (components of the elastodynamics Green's tensor)

$U_{S}^{(q)}(\bar{M}, N ; t-\tau) ; t, \tau \geq 0 ; t \geq \tau ; \bar{M} \in \Gamma_{U}$ - values of the displacements $U_{S}^{(q)}(M, N$; $t-\tau)$ on the surface $\Gamma_{U}$

$P_{S}^{(q)}(\bar{M}, N ; t-\tau)=0 ; t, \tau \geq 0 ; t \geq \tau$ - tractions created by the displacements $U_{S}^{(q)}(\bar{M}, N ; t-\tau)$ on the surface $\Gamma_{P}$

$\beta_{1} U_{S}^{(q)}(\bar{M}, N ; t-\tau)+\beta_{2} P_{S}^{(q)}(\bar{M}, N ; t-\tau)=0 ; t, \tau \geq 0 ; t \geq \tau ; \bar{M} \in \Gamma_{U P}$ - linear combination of the displacements

$U_{S}^{(q)}(\bar{M}, N ; t-\tau) ; t, \tau \geq 0 ; t \geq \tau$ and tractions $P_{S}^{(q)}(\bar{M}, N ; t-\tau)=0 ; t, \tau \geq 0 ; t \geq \tau-$ prescribed on the surface $\Gamma_{U P}$

$U_{q}(M, N ; t-\tau) ; t, \tau \geq 0 ; t \geq \tau-$ displacements in an inner point of observation $M$ in the direction of the cylindrical axis $(q \equiv \rho, \psi, \xi)$ corresponding to an inner unit point heat source applied in an inner point $N$

$U_{q N}(\widetilde{M}, N ; t-\tau) ; t, \tau \geq 0 ; t \geq \tau-$ influence functions corresponding to a unit point heat flux on the surface $\Gamma_{N}$ and representing the dynamical thermoelastic displacements

$\partial U_{q}(\widetilde{M}, N ; t-\tau) / \partial n_{\widetilde{M}} ; t, \tau \geq 0 ; t \geq \tau$ - influence functions corresponding to a unit point temperature on the surface $\Gamma_{D}$ and representing the dynamical thermoelastic displacements

$U_{q}(M, N ; t)$ - influence functions corresponding to an initial unit temperature field and representing the dynamical thermoelastic displacements

$U_{q M}(\tilde{M}, N ; t-\tau) ; t, \tau \geq 0 ; t \geq \tau-$ influence functions corresponding to a unit point heat exchange of the body through the surface $\Gamma_{M}$ and representing the dynamic thermoelastic displacements

$L^{(q)}=\delta_{q \rho} \partial / \partial \rho+\delta_{q \psi} \partial / \rho \partial \psi+\delta_{q \xi} \partial / \delta \xi$ - differential operator in cylindrical coordinates

$\nabla_{M}^{2}=\partial^{2} / \partial r^{2}+\partial / r \partial r+\partial^{2} / r^{2} \partial \varphi^{2}+\partial^{2} / \partial z^{2}$ - Laplace differential operator with respect to the cylindrical coordinates of the inner point $M$

$H_{k}-$ Heaviside function

\section{REFERENCES}

1. B. A. Boley and I. H. Weiner, Theory of Thermal Stresses, Wiley, New York, 1960.

2. A. D. Kovalencko, Fundamentals of Thermoelasticity, Naukova Dumka, Kiev, 1970. (Russian)

3. E. Melan and H. Parkus, Wärmespannungen Infolge Stationarer Temperaturfelder, Springer, Vienna, 1953.

4. V. M. Mayzel, The Temperature Problem of the Theory of Elasticity, Publisher AN SSSR, Kiev, 1951. (Russian)

5. W. Nowacki, Thermoelasticity, Pergamon Press and Polish Scientific Publishers, Oxford, Warszawa, 1962.

6. W. Nowacki, The Theory of Elasticity, Mir, Moscow, 1975. (Russian)

7. R. B. Hetnarski and M. R. Eslami, Thermal Stresses - Advanced Theory and Applications, XXXII+ 559 pages, Springer, 2009. 
8. V. D. Sheremet, The Integral Equations and Green's Matrices of the Influence Elements Method in the Mechanics of Solids, Dr. Habilitat Thesis, Technical University of Moldova, Chisinau, 1995. (Romanian)

9. V. D. Seremet, The modification of Maysel's formula in the stationary thermoelasticity, Bulletin of Academy of Science of Republic of Moldova, Mathematics, 3, Chisinau, Republic of Moldova, pp. 19-22, 1997. (English)

10. V. D. Seremet, Some New Influence Functions and Integral Solutions in Theory of Thermal Stresses, Proc. IV-th International Congress on Thermal Stresses, Osaka Prefecture University, Japan, p. 423, 2001.

11. V. D. Seremet, New Results in 3-D Thermoelasticity, Proc. 14th U.S. National Congress of Theoretical and Applied Mechanics, Blacksburg, Virginia, p. 29, 2002.

12. V. D. Seremet, Handbook on Green's Functions and Matrices, WIT Press, Southampton and Boston, 2003.

13. V. Sheremet, New Formulae for Dynamical Thermal Stresses, J. Thermal Stresses, vol. 25, no. 2, pp. 123-153, 2002.

14. V. Sheremet, Generalization of Green's Formulae in Thermoelasticity, Collection: Multiscale Green's Functions for Nanostructures, National Science Digital Library, NSF, website: http://209.85.135.104/search?q=cache:-_vI47Sx7XYJ:matdl.org/ repository/view/matdl:571, pp. 1-4, 2003.

15. V. D. Seremet, I. Vlad and A. Seremet, New Integral Formulae in Thermoelasticity, Proc. 16th ASCE Engineering Mechanics Conference (EM 2003), Washington University, Seattle, p. 82, 2003.

16. V. D. Seremet, I. Vlad, A. Seremet, New Influence Functions for Thermoelastic Sperical Shells, Proc. Vth International Congress on Thermal Stresses (ICTS 2003), Virginia Tech., Blacksburg, vol. 1, p. MA-5-1-1, 2003.

17. V. Seremet, G. Bonnet, and T. Speianu, Influence Functions and Integral Formulae for Spherical Thermoelastic Bodies, Proc. of the XXII International Congress of Theoretical and Applied Mechanics, ICTAM 2008, Adelaide University, Australia, p. 226, 2008.

18. A. D. Kartashov, Analytic Methods in the Theory of Heat Conductibility of Rigid Bodies, High School, Moscow, 1980. (Russian)

19. V. M. Budac, A. A. Samarskii and A. N. Tihonov, Set of Problems on Mathematical Physics, Gostehnizdat, Moscow, 1980. (Russian)

20. V. Seremet, Some New Results in Constructing of 3D Green's Matrices. Proc. 15th ASCE Engineering Mechanics Conference (EM 2002), Columbia University, New York, p. 91, 2002.

21. V. D. Sheremet, D. Precupan, I. Vlad and A. V. Sheremet, The Constructing of Green's Matrices in Cylindrical Coordinates, Proc. 17th ASCE Engineering Mechanics Conference (EM 2004), University of Delaware, Newark, p. 87, 2004.

22. V. Seremet, G. Bonnet, and T. Speianu, New Results in Construction of the Green's Matrices in Spherical Coordinates, Proc. of the Inaugural International Conference of the Engineering Mechanics Institute-EM08, University of Minnesota, Minneapolis, p. 240, 2008. 